\title{
Status and Development Trend of Measurement Technology about Aircraft Weight and Gravity Center
}

\author{
Wenqiang $\mathrm{Li}^{1,2, a}$, Zhenyun Duan ${ }^{1, \mathrm{~b}}$ and Zewei Yuan ${ }^{1, \mathrm{c}}$ \\ ${ }^{1}$ School of Mechanical Engineering, Shenyang University of Technology, Shenyang 110870, China \\ ${ }^{2}$ AVIC Shenyang Aircraft Corporation, Shenyang 110850 china \\ alwqsss@163.com, bduanzhenyun@126.com, zwyuan@aliyun.com
}

\begin{abstract}
Keywords: Aircraft weight; Gravity center; Flexible measuring; Development trend.
Abstract. The measurement about aircraft weight and gravity center will be used to verify theoretical weight and center of gravity. It is the most important parameters of the aircraft design and use process. There are various methods to measure the aircraft weight and gravity center. This work discusses different measuring methods and concludes the measurement trend of flexible, rapidity and precision. On the basis, this paper proposes the flexible measuring technique to meet various types of aircraft. The result shows the flexible measurement is novel technique for measuring aircraft weight and gravity center.
\end{abstract}

\section{Introduction}

The measurement about weight and center of gravity has a great significance to the design, manufacturing and use of aircraft. The changes of aircraft weight and center of gravity will affect the flying, maneuvering, rising and landing properties of aircraft. It associates with the personnel security and aircraft fly security. So it is very necessary to measure the weight and center of gravity accurately and rapidly.

The measurement of weight and gravity center is to determine the aircraft weight and gravity center, and to verify the theoretical weight and gravity center. Also the aircraft center of gravity will be relocated according to the requirements of the specific flight ${ }^{[1-2]}$. At the stage of design and assembly, the measurement of weight and gravity center must be done before the system debugging. The work is also done before and after the maintenance or modification. The transfinite weight and center serious departure will affect the normal flight. So the measurement of weight and gravity center is very important for aircraft manufacturing.

The methods widely used for measuring aircraft weight and gravity center at present include using jack, weighting platform, compound methods, etc. With modern aircraft using more and more new technology and new method, the system integration of aircraft becomes higher and higher, and the performance becomes more and more advanced. The existing measurement methods could not meet the flight safety demand of high precision, high speed, high reliability measuring condition. Therefore it is necessary to analysis and summarizes the existing measuring technology, and put forward the new measurement technology. And the basic of analyzing the existing methods and summarizing the development trend, this work propose a new flexible measuring method to meet the above demand.

\section{The measuring methods of aircraft weight and gravity center}

At present, the measurement of the weight and gravity center of widely adopts the balance principle of moment. Measurement methods include measuring jack, weighing platform, compound measure and so on. These methods have their advantages and disadvantages. Each method needs 3, 4 or more measuring points. The position of the weight and gravity center can be obtained by measuring sensor signal, collecting data, processing signal, computer calculating and converting the location into the actual aircraft coordinate system. The measurement technic, principle and method of aircraft weight gravity center could affect the measuring accuracy. The existing measuring methods are following: 
Jack measurement. Jack method is commonly used in the measurement. During measuring, the aircraft is parked on flat ground area for the machine weighing, without interference such as wind, strong magnetic field and vibration source. Regarding the hole under the aircraft as supporting point, a force measuring unit is placed between the support hole and jack sensor to percept the support force. By adjusting the three jack, the aircraft transfers to the levels, bowed their heads and look up three position. Finally the weight will be measured and gravity center will be calculated. Three-point support measurement is widely used. Fig. 1 shows the schematic diagram of jack measurement. A jack is put on the fore body as well as left and right wings. Sensor payload is the measured aircraft weight. The longitudinal position of the aircraft gravity center can be obtained by the principle of moment balance.

Usually aircraft design bases of the aircraft coordinate system. Therefore in general, the measurement results and calculation of aircraft gravity center should be given in the aircraft coordinate system. So according to the measured actual center of gravity location, aircraft gravity center is shown on the aircraft coordinate system with transforming geometric method.

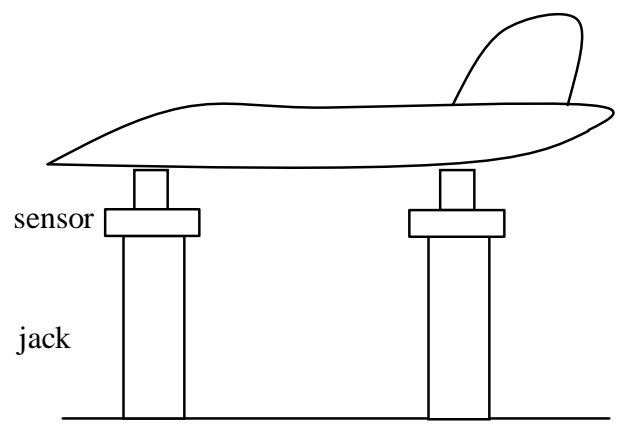

Fig.1 Schematic diagram of jack measurement

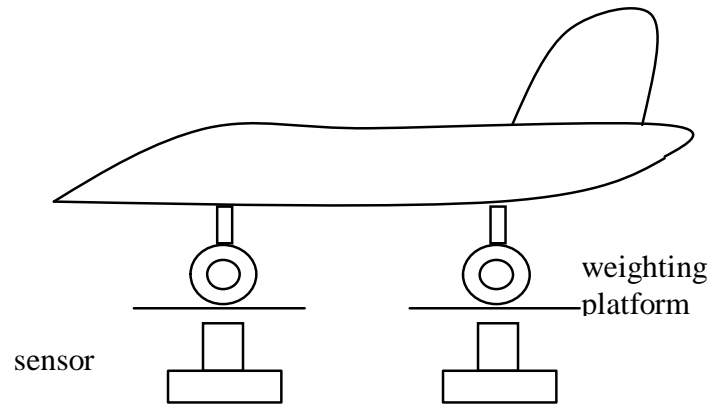

Fig.2 Schematic diagram of weighing platform

Weight measuring platform. The working principle of weight measuring platform ${ }^{[3-5]}$ is, three weighing platforms are placed under each wheel of aircraft. Each platform is placed a weighing sensor. During measuring, the aircraft is placed on weighing platform through the approach of traction. The aircraft weight goes through three weighing platforms and is detected by the sensor. The schematic diagram of weighing platforms is shown in Fig.2. According to principle of moment balance ${ }^{[6]}$, the gravity center position of aircraft is obtained by using analytical and graphical method. At last the gravity center could be calculated by transfer into aircraft coordinate system.

Due to the aircraft tire is circular, contacting force direction perpendicular to the plat ground. The component force in horizontal direction could be avoided. So the measurement error could be reduced.

Compound measuring method. In order to enhance the measuring accuracy of weighing platform, the compound measuring method combines the weighting platform with jack measurement. As shown in Fig.3, three jack are placed on each weighting platform, regarding the weighing platform as the base of jack ${ }^{[7]}$. The jack supports way could solve the problem of the level adjusting. At last, the aircraft weight could be calculated with measured force minus the jack weight.
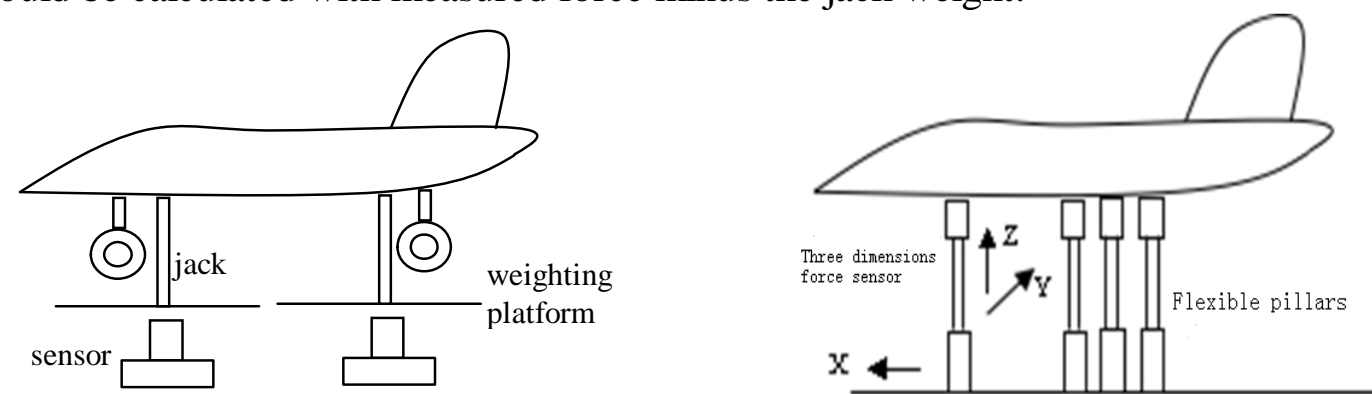

Fig.3 Schematic diagram of compound measuring method Fig.4 Flexible measuring platform

Real-time calculation measurement of weighting balance. With the need of military and the development of economic, the proportion of large aviation conveyor becomes bigger and bigger. Weighting balance system is important and necessary system for weight and center measurement of large aircraft. At present, Boeing and Airbus usually adapt the weighting balance and real-time 
calculation measurement methods. The former is used on earth, the latter is used on the fly. Both realize the automatic measurement ${ }^{[8-9]}$.

The measuring principle of ground-weighting balance system: total weight of aircraft is supported by hydraulic buffer under fuselage. Total aircraft weight consists of the pressures from each hydraulic buffer, tire's weight, brake system weight and steering beam weight. The weighting sensor is installed under the hydraulic buffer of landing gear. So the aircraft's weight could be measured exactly by accurately measure the pressure of hydraulic buffer. The position of aircraft gravity is calculated with the moment of hydraulic buffer relative to the benchmark point.

The principle of real-time measurement about weight and gravity in flying process is that: The total weight and gravity in flying process is obtained by reducing the consumed weight distribution from the known distribution of weight and gravity. Real-time calculation measurement system has been used by Boeing aircraft. During flying process, all kinds of parameters could be detected with sensors. And the aircraft weight and gravity center are timely calculated. Therefore, the pilots can master the aircraft in dynamic balance situation, and ensure the aircraft safe.

The weighting balanced system designed by Taiyuan aviation instrument co., LTD in china consists of weighting balanced computer, sensor data management and shown computer, middle pressure sensor of hydraulic buffer and hold sensor group and so on. The system has been already applied effectively.

Compare between different measuring methods. All kinds of measurement features are shown in Table1.

Table 1 The feature of different measuring methods

\begin{tabular}{ccccc}
\hline method & $\begin{array}{c}\text { structur } \\
\text { e }\end{array}$ & measurement accuracy & safety & Cost $/ \mathrm{m}$ \\
\hline jack & simple & $3-4 \%$ low & 60 \\
\hline weighting platform & complex & $2-3 \% 0$ & high & 90 \\
\hline mixing & complex & $1-2 \% 0$ & low & 130 \\
\hline real-time calculation & complex & $1 \% 0$ & high & 300 \\
\hline
\end{tabular}

(1) Jack measurement.

This system has simple structure, small size and few occupy position. It is easy to operate and move, and it is also very cheap. However, the lateral force will affect the measurement accuracy and repeatability. The out-sync in the process of lifting and the attitude angle with greater than 6 degrees will lead security issue, such as the sideslip. For large tonnage aircraft, the wings are prone to deformation because of the jack load.

(2) Weight measuring platform

For this method, lateral force has no effect on weight and gravity center. Improvement of sensor precision increases remarkably measurement accuracy. The measurement error will not change with the posture. But this method needs special fixed position and testing equipment for measuring. It also has large volume and high cost. Because of complex level-adjustment and poor coordination, it is necessary to add an appropriate plate under the wheel for level adjustment. Taking into consideration of the influence of buffer pillars friction, and the influence of aircraft attitude on the bearing of buffer pillar, the level adjustment becomes difficult.

(3) Compound measuring method

Compound method integrates the characteristics of the jack and the weighing platform. So the measurement accuracy is significantly increased. But the operability is poor, and it cannot eliminate the security problems brought by non-sync in the process of lifting aircraft.

(4) Real-time calculation measurement of weighting balance

This way adopts automatic measurement, almost does not need manual intervention. It is particularly suitable for the large tonnage aircrafts. This method effectively improves the overall 
performance of aircraft and ensures the pilot quickly master dynamic balance of aircraft to ensure the safety of flight. But the cost is higher due to its complex system.

Though there are four different methods to measure the aircraft weight and gravity center, there are some problems which need to be further improved, such as complex system structure, not high measuring accuracy, the tedious measurement process, poor measuring equipment commonality.

\section{Development trend}

Measurement is the important section in the process of manufacture. The ensured quality is the basic demand. With the development of measurement technology, the trend of measuring aircraft weight and gravity center may include: flexibility, rapidity and precision.

Flexibility. The flexibility of measurement technology about aircraft weight and center mainly embodies in the measurement equipment's flexibility. According to different type aircrafts, the supporting ways may be different. So the measurement equipment may suitable for several type aircraft. There is no need to build different measuring equipment. Flexible equipment could accomplish the measurement of multi-variety production. Research the measurement equipment which can adopt flexibility measurement for aircraft of different types and specifications. The technology will have wide adaptability and broad prospects for development.

Rapidity. Traditional measurement technology is a very complex process. The measurement period is longer, which brings up some follow-up questions such as affecting the normal aircraft's scheduling processes. Aircraft manufacturing belongs to big project. There are many difficulties in the field of aircraft location, horizontal adjustment, and gravity measurement. So it is very urgent to improve the speed of measurement.

Precision. The final purpose of measurement is to make the results precise. Measurement precision is key in the aircraft manufacture. The precision demand of aircraft weight measurement is better than $1 \%$, and the precision demand of gravity center is better than $\pm 0.5 \%$ MAC. In order to improve precision, it is needed to improve and innovate measurement methods.

\section{The flexible aircraft weight and gravity center measurement technology}

Aviation weapon equipment has the characteristics of multi-variety, small batch and rapid development. The support way is flexible and changeable in different aircrafts, so there may be three, four, or more points. The traditional measuring methods cannot meet the demands. And the measuring accuracy is not high. Enlightened by digital detection of aircraft, measurement technology research and application experience, and its flexible technique widely used in assembly fixtures in recent years, a kind of flexible weight center of gravity measurement is proposed. This method changes the traditional way of measuring and improves the technical level of the measurement.

The principle of flexible measuring weight and gravity center: Location is determined through multiple directions and adjustable flexible column, and sensors are placed on columns. Propped up by the columns, the aircraft is adjusted to level, and then the weight is measured. The position of gravity center is calculated with the moment balance principle. It ensure that the gravity center locates in the safety design range and meets the measuring requirements of different models. Flexible weight center of gravity measurement is shown in Fig.4. First, make sure how many supporting points the aircraft has. Then the columns should be adjusted to the supporting points. Three dimensions force sensor are placed on the columns. The aircraft is adjust to a level state by adjusting the columns. The movement of the pillars in the $\mathrm{X}, \mathrm{Y}$ direction is controlled by using the feedback value from the sensor to reduce effect of the lateral stress on the measurement. Actual aircraft weight data is measured with the measurement system and inputted into the data processing system. Finally, the weight and position of gravity center of aircraft will be automatically calculated by using calculation formula. Finally, the data will be displayed or printed. Therefore, the automatic measurement completes. 
This method is suitable for various types, the multi-point supporting way, and high precision measurement. Based on this principle, add returnable slider to the bottom of the jack. But this method has poor flexibility, so it is necessary to do further study to meet adaptability of various models, multi-variety weight of the center of gravity measurement.

\section{Conclusion}

(1) The aircraft weight and gravity center is premise to ensure normal flight. The enhancing measurement technology of aircraft weight and gravity center is important base for aircraft industry. This paper summarizes the technology status about measurement technology of aircraft weight and gravity center. Comparing existing measurement techniques, the paper proposes flexible measurement technology. Flexible measurement technology has a good potential application in measuring aircraft weight and gravity center.

(2) The trend of measuring aircraft weight and gravity center is flexibility, rapidity, precision. To achieve this purpose, it need to explore basic theory of measurement, break through key technique, develop precision measurement equipment and establish the technical standards for measurement.

(3)With the rapid development of digitization, computer, and automatic measuring and controlling technique, the measurement technology of aircraft weight and gravity center is also promoted. However, there are some problems in technical application. Researching the technical status and developing tendency has important significance for enhancing the level of measuring aircraft manufacturing industry.

\section{Reference}

[1] Liu Bin. Application and development of aircraft weight and center of gravity measurement techniques. Aviation maintenance and engineering Vol. 1 (2004), p.53-57.

[2] Feng Yundong. Application of weighing equipment of aircrafts. Measurement and Testing. (2011), p.250-251.

[3] Jiang Dianxiang, Liu Zhuo, Lin Ziping. Research aircraft center of gravity of the weight measurement methods. Trainer, Vol.3 (2011), p.47-52.

[4] Liu Desheng, Research aircraft weight, center of gravity measurement and calculation methods. China Aviation Society 2008 Annual Conference, p.144-147.

[5] Pan Nuogang. Explore the Whole machine precision gravity measurement methods. Aircraft Design, Vol.30 (4), p.12-15.

[6] Chen Junzhang, Liu Mengzhao, Sun Zhenjia. Aircraft Design Manual Volume VIII. Beijing: Aviation Industrial Publishing House, 1999.

[7] Jiang Dianxiang, Liu Zhuo. Measurement the center of gravity of a certain type of machine. China Aviation Society Weight Engineering Academic Exchange In 2006.

[8] Xuan Xiaogang, Yao Minqiang. The application of weighing balance system in large aircraft weight center of gravity measurement. Aviation Industry Technology, Vol.22 (2008), p.53-55.

[9] Luo Gang. Aircraft weight centered on real-time analysis system, China Manufacturing Automation, Vol.2 (2011), p.50-51. 